\title{
Josephson Junctions as Threshold Detectors of the Full Counting Statistics: Open issues
}

\author{
Tomáš Novotný \\ Department of Condensed Matter Physics, Faculty of Mathematics and Physics, \\ Charles University, Ke Karlovu 5, 12116 Prague, Czech Republic \\ E-mail: tno@karlov.mff.cuni.cz
}

\begin{abstract}
I study the dynamics of a Josephson junction serving as a threshold detector of fluctuations which is subjected to a general non-equilibrium electronic noise source whose characteristics is to be determined by the junction. This experimental setup has been proposed several years ago as a prospective scheme for determining the Full Counting Statistics of the electronic noise source. Despite of intensive theoretical as well as experimental research in this direction the promise has not been quite fulfilled yet and I will discuss what are the unsolved issues. First, I review a general theory for the calculation of the exponential part of the non-equilibrium switching rates of the junction and compare its predictions with previous results found in different limiting cases by several authors. I identify several possible weak points in the previous studies and I report a new analytical result for the linear correction to the rate due to the third cumulant of a non-Gaussian noise source in the limit of a very weak junction damping. The various analytical predictions are then compared with the results of the developed numerical method. Finally, I analyze the status of the so-far publicly available experimental data with respect to the theoretical predictions and discuss briefly the suitability of the present experimental schemes in view of their potential to measure the whole FCS of non-Gaussian noise sources as well as their relation to the available theories.
\end{abstract}

\section{Introduction}

Josephson junctions (JJs) were proposed as threshold detectors of the Full Counting Statistics (FCS) by Tobiska and Nazarov [1] and independently by Pekola [2] in 2004. Since then there has been continuing effort to implement the proposed schemes experimentally as well as to improve them and better understand their potential theoretically. The original scheme by Tobiska and Nazarov 1] proposed using overdamped JJ as the threshold detector. This appears to be problematic since in the overdamped junction when the effective phase particle overcomes the tilted washboard potential barrier it gets immediately retrapped in the adjacent minimum. This results in the phase diffusion which, however, does not yield enough sensitivity for detecting the whole FCS. This could in principle be overcome by employing a negative-inductance device which apparently hasn't appealed to the experimentalists enough to actually implement it. Instead they opted for an obvious alternative to use underdamped junctions where, under suitable conditions, once the particle overcomes the first barrier it keeps on sliding down the potential thus producing finite voltage. Thus, the switching of an underdamped junction between the supercurrent (static 
phase) and running (finite phase velocity, i.e. finite voltage) state would provide a prime example of a threshold detector. Unfortunately, this innocently-looking change in the setup dramatically changes the level of difficulties involved in the theoretical analysis. This paper addresses those difficulties in some detail.

The structure of the paper is the following. In the next section 2 I report the theoretical concept of calculating the non-equilibrium escape rate due to a nonGaussian noise source whose FCS is to be determined. The general theory based on the WKB-like approximation for the weak noise intensity is further carried out to an analytical result in case of the linear perturbation theory in the third cumulant for very weak junction damping in subsection 2.1. In this subsection I also make comparison with alternative existing theories. In the next subsection 2.2 the full theory is numerically implemented and the numerical results in an experimentally relevant regime are discussed and further compared with various analytical predictions. In section 3 I briefly raise some experimentally relevant questions such as what is the effect on the rate asymmetry of the nominally subleading terms entering the rate and whether one can actually experimentally leave the linear regime and achieve the measurement of the whole FCS of a noise source. In the last section 4 I summarize what has been achieved in this work and review the remaining open problems.

\section{Theoretical calculation of the non-equilibrium escape rate}

The Josephson element in an electrical circuit is often modeled as a current biased $\left(I_{b}\right)$ resistively $(R)$ and capacitively $(C)$ shunted ideal JJ with the Josephson currentphase relation $I_{J}(\varphi)=I_{0} \sin \varphi$. The voltage across the junction is determined by the second Josephson relation $V_{J}=\dot{\varphi} \hbar / 2 e$ with the time-derivative denoted by the dot. Moreover, due to the action of ubiquitous thermal (Gaussian) noise $\xi(t)$ characterized by the temperature $T$ and non-equilibrium electronic noise $\eta(t)$ from the measured device whose FCS is to be determined, the JJ is subjected to stochastic forces and its dynamics is thus described by the following Langevin equation (RCSJ model)

$$
\ddot{\varphi}+\frac{1}{R C} \dot{\varphi}+\frac{2 e}{C \hbar}\left(I_{0} \sin \varphi-I_{b}\right)=\xi(t)+\eta(t) .
$$

In a realistic experimental situation the current-bias assumption can be inadequate and one may need to generalize the above model. The general consequences of an imperfect current bias are so called environmental or "cascade" corrections to the measured cumulants of the source FCS which were studied in previous works [3, 4. They could be straightforwardly included here in the same spirit as in those works, especially Ref. 4, but since they appear to be of minor importance in the so far reported experiments I will neglect them.

In this study I will consider in detail exclusively the simplest case of the Poissonian shot noise $\eta(t)$ corresponding to the measured device being a tunnel junction. In such a case $\eta(t)$ is just a train of $\delta$-function-like spikes which are separated by an exponentially distributed waiting time with a single parameter (mean waiting time) being the mean (particle) current $I_{m} / e$ flowing through the tunnel junction. This case is also the only one studied experimentally by this type experiments to date. Assuming the temporal width of the pulses composing $\eta(t)$ to be very small compared to a characteristic time of the junction dynamics (which is its plasma frequency $\omega_{p 0}=\sqrt{2 e I_{0} / \hbar C}$ ) one can obtain a master equation (analogous to the Fokker-Planck equation in case of Gaussian noise only) for the probability density $W(x, v, t)$ in dimensionless units 


$$
\begin{aligned}
t \omega_{p} \rightarrow t, \varphi \rightarrow x, \dot{\varphi} / \omega_{p 0} & \rightarrow v \\
\frac{\partial W}{\partial t} & =-v \frac{\partial W}{\partial x}+Q^{-1} \frac{\partial(v W)}{\partial v}+\left(\sin x-s+\frac{I_{m}}{I_{0}}\right) \frac{\partial W}{\partial v}+Q^{-1} \frac{k_{B} T}{E_{J}} \frac{\partial^{2} W}{\partial v^{2}} \\
& +\frac{I_{m}}{I_{0} \lambda}\left[\exp \left(-\lambda \frac{\partial}{\partial v}\right)-1\right] W
\end{aligned}
$$

with $s=I_{b} / I_{0}$ the rescaled bias current, $Q=R C \omega_{p 0}$ the quality factor of the (unbiased 国) junction and $\lambda=\sqrt{e^{2} / C E_{J}}$ with the Josephson energy of the junction proportional to the critical current $E_{J}=I_{0} \hbar / 2 e$. The last term in the equation can be identified as stemming from the cumulant generating function of the Poissonian process $F_{\text {Poisson }}(x)=I_{m}(\exp x-1)$ [ which suggests how to deal with non-Poissonian noise source provided the Markovian approximation is made. Thus, the substitution $I_{m}[\exp (-\lambda \partial / \partial v)-1] \rightarrow F(-\lambda \partial / \partial v)$ for general noise sources described by the cumulant generating function $F(x)$ generalizes the particular results shown here for the Poissonian process to arbitrary noise sources as long as the Markovian approximation is justified [1, 5].

In order to calculate the escape rates of the junction from the supercurrent branch (zero voltage state with a static phase $\varphi$ ) to the running state (finite voltage across the junction with non-zero phase velocity $\dot{\varphi}$ ) in the low noise limit we use the standard technique known as the singular perturbation theory in the mathematical literature [6] or as WKB method in the physical context [7, 8]. It consists in making the ansatz $W(x, v, t)=\exp [S(x, v, t) / \theta]$ for the probability density $W(x, v, t)$ with $\theta$ being a small parameter related to the noise intensity: $\theta \equiv k_{B} T_{\text {eff }} / E_{J} \equiv k_{B} T / E_{J}+Q \lambda I_{m} / 2 I_{0}=$ $k_{B} T / E_{J}+e R I_{m} / 2 E_{J}$. Thus, $\theta$ is a dimensionless effective temperature of the junction due to the summed effect of the thermal noise and the Gaussian part of the nonequilibrium noise [1, 3, 5, 9, 10. When this ansatz is put into Eq. (2) and the lowest order in $\theta$ is only retained (corresponding to the WKB approximation and justified for small $\theta \ll 1$ ) we obtain the following Hamilton-Jacobi (HJ) equation, i.e. a first order partial differential equation for $S(x, v, t)$

$$
\begin{aligned}
\frac{\partial S}{\partial t} & =-v \frac{\partial S}{\partial x}+(\sin x-s) \frac{\partial S}{\partial v}+Q^{-1} v \frac{\partial S}{\partial v}+Q^{-1}\left(\frac{\partial S}{\partial v}\right)^{2}+\frac{I_{m}}{I_{0}} \sum_{n=3}^{\infty} \frac{1}{n !}\left(\frac{\lambda}{\theta}\right)^{n-1}\left(-\frac{\partial S}{\partial v}\right)^{n} \\
& =-v \frac{\partial S}{\partial x}+(\sin x-s) \frac{\partial S}{\partial v}+Q^{-1} v \frac{\partial S}{\partial v}+Q^{-1}\left(\frac{\partial S}{\partial v}\right)^{2}+\frac{\theta}{I_{0} \lambda} \tilde{F}_{\text {Poisson }}\left(-\frac{\lambda}{\theta} \frac{\partial S}{\partial v}\right)
\end{aligned}
$$

For a general noise source the last term (given by the sum) in the preceding equations would be replaced by the corresponding expression $\tilde{F}(x)=F(x)-F^{\prime}(0) x-F^{\prime \prime}(0) x^{2} / 2$, i.e. by the reduced cumulant generating function with the first two moments (mean current and the zero-frequency noise) subtracted (notice that $F(0)=0$ by definition).

This Hamilton-Jacobi equation can be solved via the method of characteristics, i.e. one can recast the equation as a dynamical system in a 4-dimensional phase-space $[x(t), v(t), p(t) \equiv \partial S / \partial x, y(t) \equiv \partial S / \partial v]$ governed by the auxiliary Hamiltonian

$$
\mathcal{H}=v p-(\sin x-s) y-Q^{-1} y(v+y)+\frac{\theta}{I_{0} \lambda} \tilde{F}\left(-\frac{\lambda}{\theta} y\right) .
$$

$\ddagger$ Note that my definition of the quality factor differs from that in Ref. [5] where a bias-specific quality factor is used instead.

$\S$ Here I assume $I_{m} \geq 0$ and the Eq. (2) holds for the positive polarity of the tunneling current. The opposite polarity would just change the sign in the exponential in Eq. (2). 
The coordinates $x, v$ and their conjugated momenta $p, y$ are then evolving according to the following equations of motion

$$
\begin{aligned}
& \dot{x}=\frac{\partial \mathcal{H}}{\partial p}=v, \dot{p}=-\frac{\partial \mathcal{H}}{\partial x}=y \cos x, \dot{y}=-\frac{\partial \mathcal{H}}{\partial v}=-p+Q^{-1} y, \\
& \dot{v}=\frac{\partial \mathcal{H}}{\partial y}=-(\sin x-s)-Q^{-1}(v+2 y)-\frac{1}{I_{0}} \tilde{F}^{\prime}\left(-\frac{\lambda}{\theta} y\right) .
\end{aligned}
$$

The exponential part of the non-equilibrium escape rate is in this language determined by the difference of the stationary, i.e. time-independent, action between the barrier top and the metastable minimum of the tilted washboard potential, i.e. $\theta \log \Gamma \propto$ $S\left(x_{\max }, 0\right)-S\left(x_{\min }, 0\right)$ [7] which can be either determined by the direct solution of Eq. (3) or by finding the action along the trajectory of the system (51) connecting in infinite time (corresponding to the stationary solution and zero auxiliary energy $\mathcal{H}=0)$ the two fix points $\left[x_{\min }, 0,0,0\right]$ and $\left[x_{\max }, 0,0,0\right]$ [11]. I will demonstrate both methods in the next two subsections. At this point I would like to stress that the overdamped analog of the present problem (with $C \rightarrow 0$ in Eq. (1) when the inertial term $\ddot{\varphi}$ can be neglected) studied in Ref. $[1$ is integrable since analogous equations to (3), (5) are to be solved in a 2-dimensional phase-space only and with the help of the stationarity constraint $\mathcal{H}_{\text {overdamped }}(x, p)=0$ one easily finds the action in Eq. (3) by a quadrature for an arbitrary strength of the non-equilibrium noise. Unfortunately, this property does not carry over to the underdamped case where the energy constraint is not sufficient for integrability. Therefore, the underdamped problem is conceptually far more difficult than the originally suggested overdamped model.

\subsection{Linear perturbation theory of the rate asymmetry due to a weak third cumulant}

In this subsection I will present a linear perturbation theory of the rate asymmetry which is an alternative to the similar previous approaches by a number of authors 3, 4, 5. I will use this limiting case for the illustration of the general method, which will be fully developed in the next subsection, and, at the same time, for pointing out possible discrepancies in the previous studies. As a by-product I will present a new analytical formula for the rate asymmetry in the very low damping limit $Q \rightarrow \infty$.

Following the previous studies we consider the linear correction to the escape rate due to a weak third cumulant. To this end we truncate the sum in the stationary version of the HJ equation (3) to the first order, i.e. we consider the effects of the third cumulant $c_{3}$ only. Further, we formulate the linear perturbation theory for an arbitrary potential $V(x)$ in which the effective particle moves - the present case is then recovered by the choice of the tilted washboard potential describing the JJ (in dimensionless units) $V(x)=-\cos x-s x$. The resulting HJ then reads

$$
0=-v \frac{\partial S}{\partial x}+V^{\prime}(x) \frac{\partial S}{\partial v}+Q^{-1} v \frac{\partial S}{\partial v}+Q^{-1}\left(\frac{\partial S}{\partial v}\right)^{2}-c_{3}\left(\frac{\partial S}{\partial v}\right)^{3},
$$

with $c_{3}=I_{m} \lambda^{2} / 6 I_{0} \theta^{2}$. We solve this equation in the linear order in $c_{3}$ by linearizing the equation. After inserting $S(x, v)=S_{0}(x, v)+c_{3} S_{1}(x, v)$ into the equation, using the knowledge of the zeroth order solution $S_{0}(x, v)=-v^{2} / 2-V(x)$ corresponding to the Boltzmann factor due to the thermal Gaussian noise, and keeping only the linear terms in $S_{1}$ one obtains

$$
v^{3}=v \frac{\partial S_{1}}{\partial x}+\left[Q^{-1} v-V^{\prime}(x)\right] \frac{\partial S_{1}}{\partial v} .
$$


It is very unlikely that Eq. (7) could be solved analytically for general $Q$. Ankerhold 5. did find certain solution to the problem of the rate asymmetry for any $Q$, however, his solution is not a solution of the above equation (7) as I will discuss later on. Indeed, one should not expect finding an explicit analytical solution to Eq. (7) for arbitrary $Q$ since it is generally known that the action $S(x, v)$ (or the "non-equilibrium potential") develops a dense set of singularities close to the barrier top [12. This does not happen only in the integrable cases which is certainly the limit $Q \rightarrow 0$ corresponding to the one-dimensional spatial diffusion and, hopefully, also for $Q \rightarrow \infty$ describing the energy diffusion limit, which is effectively one-dimensional again.

Here, I give an analytic expression for the solution $S_{1}(x, v)$ in the limit $Q \rightarrow \infty$ for general potential $V(x)$, in particular for the tilted washboard potential without resorting to its cubic approximation employed in previous works [5, 4. We look for the solution of Eq. (77) with $Q \rightarrow \infty$ in the form $S_{1}(x, v)=\phi_{0}(x)+\phi_{2}(x) v^{2} / 2$ and find a closed set of equations

$$
\begin{aligned}
\phi_{2}^{\prime}(x) & =2, \\
\phi_{0}^{\prime}(x) & =V^{\prime}(x) \phi_{2}(x) .
\end{aligned}
$$

The solution $\phi_{2}(x)=2\left(x-x_{0}\right), \phi_{0}(x)=2 \int^{x} d y y V^{\prime}(y)-2 x_{0} V(x)+C$ contains two arbitrary constants $C, x_{0}$. Moreover, one can add an arbitrary solution of the homogeneous part of Eq. (7) to this particular solution. Solutions to the homogeneous problem are arbitrary (sufficiently smooth and differentiable) functions of the particle energy $G\left(v^{2} / 2+V(x)\right)$, thus, the freedom in the particular solution can be absorbed into the homogeneous solution since it just represent a linear function of the energy. The arbitrariness stemming from the mathematical solution must be fixed by physical requirements. First, all physical quantities must be "gauge invariant" meaning that an arbitrary constant shift in the potential $V(x) \rightarrow V(x)+\Delta$ cannot change the physical observables. Those are changes of $S_{1}(x, v)$ between different points in the phase-space, i.e. not just $S_{1}(x, v)$ itself, rather its partial derivatives $\partial S_{1} / \partial x$ and $\partial S_{1} / \partial v$. The conditions to be satisfied are then $\partial^{2} S_{1} / \partial x \partial \Delta=0$ and $\partial^{2} S_{1} / \partial v \partial \Delta=0$. Both lead to the same equation $G^{\prime \prime}(x)=0$ with the linear function solution. This way, we recover the freedom stemming from the particular solution but the larger freedom of the homogeneous solution has been removed. The remaining uncertainty, being basically just the choice of the origin of integration $x_{0}$, since the constant $C$ is harmless, is fixed by the requirement that in the vicinity of the potential minimum, where the potential can be approximated as harmonic, all the Gaussian averages (i.e. $\langle v\rangle,\langle x\rangle,\left\langle x^{2}\right\rangle,\langle x v\rangle,\left\langle v^{2}\right\rangle$ ) of the original Fokker-Planck/master equation must stay intact by the third cumulant. In the harmonic regime, this is a necessary consequence of the linearity of the underlying Langevin equation. This condition implies that the origin of integration must be identical with the potential minimum $x_{0}=x_{\min }$. In total, one finally has

$$
S_{1}(x, v)=2 \int_{x_{\min }}^{x} d y\left(y-x_{\min }\right) V^{\prime}(y)+\left(x-x_{\min }\right) v^{2}+C,
$$

yielding for the exponential part of the rate asymmetry $R_{\Gamma} \equiv \Gamma_{+} / \Gamma_{-}$(factor of 2 stands for the sum of the two equal contributions to the asymmetry from the two opposite polarities of the measured current) $R_{\Gamma}(Q \rightarrow \infty)=\exp \left[2 c_{3}\left(S_{1}\left(x_{\max }, 0\right)-\right.\right.$ $\left.\left.S_{1}\left(x_{\min }, 0\right)\right) / \theta\right]=\exp \left[2 D_{1}(s) E_{J}^{2} \ll I_{m}^{3} \gg / C I_{0}\left(k_{B} T_{\text {eff }}\right)^{3}\right]$ with the function $D_{1}(s)$ introduced in Ref. [3] reading

$$
D_{1}(s) \equiv \frac{1}{6}\left[S_{1}\left(x_{\max }, 0\right)-S_{1}\left(x_{\min }, 0\right)\right]=\frac{1}{3} \int_{x_{\min }}^{x_{\max }} d x\left(x-x_{\min }\right) V^{\prime}(x)
$$




$$
\begin{aligned}
& =\frac{1}{3} \int_{\arcsin s}^{\pi-\arcsin s} d x(x-\arcsin s)(\sin x-s) \\
& =\frac{2}{3} \arccos s\left[\sqrt{1-s^{2}}-s \arccos s\right]
\end{aligned}
$$

where the second part applies to the particular case of the tilted washboard potential.

The value of $D_{1}(s)$ at zero is $D_{1}(0)=\pi / 3$ in accordance with Ref. [3] while the asymptotics for large bias is $D_{1}(s \rightarrow 1) \approx a(1-s)^{2}$ with $a=8 / 9$ which is exactly equal to the result by Ankerhold [5] but it actually differs from SJ's numerical finding $a \approx 0.8$ 3 further supported by an independent study by Grabert [4] with $a \doteq 0.79$. Although the difference is not severe being on the order of $10 \%$ only and, therefore, practically most likely irrelevant, from the conceptual point of view it matters because all the works claim to calculate the same quantity for the very same model and, thus, the correct result should be unique. It's not simple to follow and reproduce SJ's approach but Grabert's method is very transparent and I have fully recovered his numerical findings. Minor generalization of his approach to the full tilted washboard potential (Grabert uses the usual cubic approximation to the tilted-washboard potential for a large bias $s \rightarrow 1$ ) gives $D_{1}(0)=\pi / 3$ (within numerical precision) and $a \doteq 0.79$ for $s \rightarrow 1$. The discrepancy with the above analytical result is thus not a problem of the numerical precision of works [3, 4] but a conceptual problem. Since Grabert uses the trajectory approach of Eq. (5D) I will put off the discussion of his work to the next subsection where the same formalism is also used.

Ankerhold [5] was looking for the correction $S_{1}(x, v)$ in the form $S_{1}(x, v)=$ $\phi_{0}(x)+\sum_{n=1}^{3} v^{n} \phi_{n}(x) / n$ and got certain conditions for the arbitrary functions $\phi_{n}(x)$ 's from the leading order solution of the Fokker-Planck equation accounting for the noise with the nonzero third cumulant. When his ansatz is plugged into Eq. (7) one can easily find that the set of equations obtained for $\phi_{n}(x)$ 's is internally inconsistent (suggesting that the truncation at the third power in $v$ in the ansatz is insufficient) for a general $Q$ and potential $V(x)$. There are several exceptions when the inconsistencies are removed, namely for a strictly harmonic potential $V(x) \propto x^{2}$ (this potential doesn't exhibit a barrier, at least not a smooth one approximating the tilted washboard potential) and in the limits either $Q \rightarrow 0$ or $Q \rightarrow \infty$. This suggest that Ankerhold's solution [5] could yield correctly the two limiting cases $Q \rightarrow 0, \infty$ for the considered cubic approximation to the potential. Indeed, in the limit $Q \rightarrow \infty$ his solution is equal to mine for $s \rightarrow 1$ as already mentioned above The opposite limit $Q \rightarrow 0$ is simple since that case is integrable for any strength of the third cumulant and the linear response can be easily calculated analytically yielding $R_{\Gamma}(Q \rightarrow 0)=\exp \left[2 D_{2}(s) 2 e R^{2} E_{J}^{2} \ll I_{m}^{3} \gg / \hbar\left(k_{B} T_{\text {eff }}\right)^{3}\right]=$ $\exp \left[2 D_{2}(s) Q^{2} E_{J}^{2} \ll I_{m}^{3} \gg / C I_{0}\left(k_{B} T_{\text {eff }}\right)^{3}\right]$ with $D_{2}(s)=\left[\left(1+2 s^{2}\right) \arccos s-\right.$ $\left.3 s \sqrt{1-s^{2}}\right] / 6 \approx 8 \sqrt{2} / 45(1-s)^{5 / 2}$ for $s \rightarrow 1$ [3, 4]. This is identical to Ankerhold's solution in the corresponding limit $Q \rightarrow 0, s \rightarrow 1$ (recall the multiplicative correction factor of $2 / 3$ in the Erratum and Ankerhold's definition of the bias-dependent quality factor of the JJ $\left.Q(s)=Q\left(1-s^{2}\right)^{1 / 4}\right)$. Now, Ankerhold's solution can be interpreted as a simple interpolation formula between the two limiting cases reading

\| Of course, this is not too surprising in view of the above-stated general properties of the nonequilibrium action, see the comments below Eq. (7).

व This fact is also not surprising since the ansatz used in my solution is just a subset of his form of $S_{1}(x, v)$. The main difference is that I used the ansatz only in the case where it does solve Eq. (7) and also the discussion of fixing the freedom in the solution due to the homogeneous part etc. (present for any Q) seems absent in his work. 
$R_{\Gamma}(Q)=\exp \left[2 D(s, Q) E_{J}^{2} \ll I_{m}^{3} \gg / C I_{0}\left(k_{B} T_{\text {eff }}\right)^{3}\right]$ with the interpolating function $D(s, Q)=D_{2}(s) Q^{2} /\left[1+Q^{2} D_{2}(s) / D_{1}(s)\right]=8 / 9 Q^{2}(s)(1-s)^{2} /\left[Q^{2}(s)+5\right]$. It turns out that Ankerhold's expression (Eq. (13) of Ref. [5]) is a neat interpolation scheme between the highly underdamped and overdamped junction limits. It certainly provides a very efficient and quite precise interpolation formula for a finite $Q$. Its detailed comparison with the numerically exact solution will be shown in the next subsection.

\subsection{Numerical evaluation of the escape rate in a general situation}

Now, we turn to the general case of the calculation of the rate asymmetry for an arbitrary intensity of the non-equilibrium noise acting on the junction. This is achieved by the numerical solution of the effective dynamical system equations (5). As already mentioned the solution consists in finding a trajectory satisfying the equations of motion (5) and connecting in infinite time (corresponding to the zero auxiliary energy $\mathcal{H}=0)$ the two fix-points $\left[x_{\min }, 0,0,0\right]$ and $\left[x_{\max }, 0,0,0\right]$ being the (metastable) minimum of the potential and the top of the barrier, respectively. There always exists a classical, "relaxation" solution corresponding to the dissipative but noise-free motion of the effective particle from the barrier top down to the minimum. This solution has $p(t) \equiv 0, y(t) \equiv 0$ and also the associated action is zero. On the other hand we are interested in the other, "escape" solution connecting the two potential extrema via trajectory with non-zero conjugated momenta $p(t), y(t)$. For equilibrium, i.e. Gaussian, noise the two types of trajectories are connected by (generalized) timereversal which forms the basis of the Onsager-Machlup theory and was used by Grabert 4. for his linear response calculations. For general non-equilibrium noise sources, however, the two trajectories are not simply related and one has to calculate the escape trajectory directly by solving the full system (5).

This is exactly done here. The problem is formulated as a boundary value problem (BVP) on an infinite time interval reflecting the stationarity condition of the original escape problem. Obviously, this makes the BVP rather tricky and one has to be cautious in its solution. Once the solution $[x(t), v(t), p(t), y(t)]$ is found the action difference between the two fix-points is calculated from the definition as

$$
\begin{aligned}
\Delta S & \equiv S\left(x_{\max }, 0\right)-S\left(x_{\min }, 0\right)=\int_{-\infty}^{\infty} d t[p(t) \dot{x}(t)+y(t) \dot{v}(t)-\mathcal{H}(x(t), v(t), p(t), y(t))] \\
& =-\int_{-\infty}^{\infty} d t\left\{Q^{-1} y(t)^{2}+\frac{\theta}{I_{0} \lambda}\left[\tilde{F}\left(-\frac{\lambda}{\theta} y(t)\right)-\tilde{F}^{\prime}\left(-\frac{\lambda}{\theta} y(t)\right)\left(-\frac{\lambda}{\theta} y(t)\right)\right]\right\} . \quad(10)
\end{aligned}
$$

The second line was obtained after using the explicit expressions for $\dot{x}(t), \dot{v}(t)$ from Eq. (5) and $\mathcal{H}$ (4) and the resulting action is thus expressed solely via the conjugate momentum $y(t)$. The exponential part of the rate asymmetry $\log \left(\Gamma_{+} / \Gamma_{-}\right)$is then calculated as the difference of the action for two opposite measured current polarities.

Technically the BVP is formulated on a long, but finite time interval estimated by the shooting solution used for the initial guess, for details see below. At the ends of this time interval the trajectory is assumed to be close enough to the respective fixpoint so that the linear approximation to the equations of motion (5) can be employed. The linearized system is then characterized by the stability analysis which identifies stable/unstable directions and corresponding eigenvalues. The boundary conditions at the ends of the time interval are then formulated with help of the respective linearized system in the spirit of the study [13, Sec. IV, i.e. the 2-dimensional unstable (stable) 
manifold around the minimum (maximum) is identified and the solution is required to lie in them which yields two boundary conditions at each fix-point. Solution of the BVP is then sought for by a relaxation method with the 'bvp4c' built-in solver in Matlab [14.

The solver needs a very good initial guess for the solution to converge at all. When it does, it is very fast and efficient. Without a good initial guess it usually does not converge, especially for very large $Q$. Thus, the initial guess is the crucial part of the whole solution process. For finding a good initial guess I solve the BVP by the shooting method first (in line with a general BVP strategy [14, 15), i.e. I look for a solution of Eq. (5) by solving an initial value problem starting at the unstable manifold around the minimum and searching for such an initial condition which evolves into the other fix-point around the potential barrier. Finding a solution by the shooting method solely is a rather difficult task due to involved characteristic numerical instabilities around the target fix-point [14, 16. Indeed, the found solution is typically not precise enough to be of any use for the evaluation of the rates, however, it usually suffices as a good enough initial guess to ensure the convergence of the relaxation method. Moreover, the solution found by the shooting method gives a good estimate for the time needed to join the two linearized manifolds around the respective fix-points, a task which is not obvious how to accomplish otherwise. After solving the problem at this fixed long time interval I include corrections due to the rests of the infinite time interval at the beginning and the end, respectively, by analytically evaluating the associated quadratic action exactly analogously to the method of Ref. [13, Sec. V. These corrections, although relatively small, are necessary within the precision required by the problem. The last technical detail concerns the handling of the (non)uniqueness of the solution. Since the system (5) is autonomous it has an infinite number of solutions related by a simple time-translation, i.e. if $[x(t), v(t), p(t), y(t)]$ is a solution then for an arbitrary $\tau$ shift in time $[x(t+\tau), v(t+\tau), p(t+\tau), y(t+\tau)]$ is also a solution. This liberty may confuse the relaxation solver and, thus, it is advisory to fix the solution by explicit breaking of the time invariance 14. I achieved this "locally" by fixing the phase of the oscillatory solution at the unstable manifold around the potential minimum both in the boundary conditions as well as in the initial value problem. Time shift by the period of the local oscillations remains to be a symmetry operation but the continuous symmetry with an arbitrary shift is this way broken. This trick does help to stabilize the solution, nevertheless, despite of all the tricks used the numerical implementation is still not absolutely stable and one can occasionally run into problems of non-convergence, especially with increasing value of $Q$. This is not so surprising since for large $Q$ the time span needed to connect the two linearized neighborhoods of the fix-points becomes longer and the solution in between exhibits ever increasing number of oscillations.

Further, due to the linear regime in which the problem is to be solved to describe current experiments the effect of non-Gaussian noise leads only to very small asymmetry effects and this requires rather high precision of the calculations which stretches the used method to its limits. Further improvement of the numerical method is thus an interesting and urging open issue in the solution of the present problem. During the UPoN2008 conference I became aware of the work by the Lancaster group [13, 17, 18, which has apparently developed a toolbox of methods for tackling even far more difficult problems of escape. While I developed some of the techniques they use independently, there seem to be some more left to explore. It will be interesting to see whether those techniques, such as the action plot concept [13], will be successful 
in improving the present numerics. My naive fast implementation attempts have failed thus far. The main difference of the present problem from most established techniques, including the Lancaster group's ones, seems to lie in the presence of nonGaussian noise and the task to actually employ and characterize its effects on the escape characteristics. It's unclear at this point how seriously this fact influences the feasibility and/or performance of those techniques developed predominantly for Gaussian problems.

For this moment I can only present numerical results obtained with the "notyet-quite perfect" BVP method described above. The results are shown in Figs. 1, 2, for parameters motivated by the Saclay experiment by B. Huard et al. [10. Their junction was characterized by the critical current $I_{0} \doteq 0.48 \mu \mathrm{A}$ equivalent to the Josephson energy $E_{J} / k_{B} \doteq 11.4 \mathrm{~K}$, quality factor $Q \doteq 22$, and the dimensionless "kick" parameter $\lambda \doteq 0.002$. The corresponding plasma frequency of the unbiased junction was $\omega_{p 0} \approx 1 \mathrm{GHz}$. These experimental parameters correspond exactly to Fig. 1 while in Fig. 2 I just modified the value of the quality factor $Q=4$ "by hand" (corresponding to changing the value of the resistance $R$ while keeping the other parameters constant) to explore a more intermediate regime of $Q$ and see the performance of different theories also there, not only in the high $Q \rightarrow \infty$ limit as in Fig. 1. The experiments were performed for temperatures in the range $20-530 \mathrm{mK}$. This is reflected by the lowest temperature of $T=20 \mathrm{mK}$ used in Fig. 1 while Fig. 2 presents results for an intermediate temperature of $T=200 \mathrm{mK}$. Compared with the Josephson coupling energy $E_{J}$ both the reservoir as well as effective temperatures are small, on the order of few per-cents, which justifies the usage of the above developed theory of the non-equilibrium action valid for the weak noise only.

The fast inspection of both figures reveals that the logarithm of the asymmetry $\log \left(\Gamma_{+} / \Gamma_{-}\right)$presented there is generally a small quantity with a typical magnitude of about $10 \%$. This is consistent with the usage of the linear response theories (in the third cumulant) employed in previous studies $3,4,5$. The curves are not, however, linear functions of the measured current $I_{m}$ due to the fact that the current contributes also to the effective temperature (and it is an important contribution) which enters the formula for the asymmetry linear in the third cumulant (proportional to $I_{m}$ as well for the tunnel junction). Moreover, there is another source of nonlinearity in the curve, namely the fact that experimentalists for convenience measure in the range of roughly constant mean escape rate on the order of $\approx 30 \mathrm{kHz}$. Thus, for each value of the measured current $I_{m}$ the (dimensionless) bias current $s$ is adjusted in such a way that the mean rate stays constant close to that value. For the present junction with $\omega_{p 0} \approx 1 \mathrm{GHz}$ this implies fixing the dimensionless barrier height to a value of roughly 10.4. In other words, for every $I_{m}$ the value of $s\left(I_{m}\right)$ is determined from the equation $\Delta U\left(s\left(I_{m}\right)\right) / k_{B} T_{\text {eff }}\left(I_{m}\right) \approx-\log \left(3 \cdot 10^{-5}\right) \doteq 10.4$ with $\Delta U(s)=2 E_{J}\left(\sqrt{1-s^{2}}-s \arccos s\right) \approx 4 \sqrt{2} E_{J}(1-s)^{3 / 2} / 3$ for $s \rightarrow 1$ being the barrier height of the tilted washboard potential and $T_{\mathrm{eff}}\left(I_{m}\right)=T+e R I_{m} / 2 k_{B}$ the effective temperature.

Let us first discuss Fig. 1 with high $Q=22$. This value of the quality factor was nearly at the edge of stability of my BVP numerics described above. The diagnostic quantities are shown in the plot to exemplify the precision achieved in the calculations. I plot the quantity $S(0)-S_{\text {anal }}(0)$ to assess the overall precision of the calculation. By $S(0)$ I denote the action calculated numerically for zero measured current $I_{m}=0$. This value is known analytically and equals the already discussed experimental value of $\approx 10.4$. This quantity, in the plot labeled ' $I_{m}=0$ ' is shown 


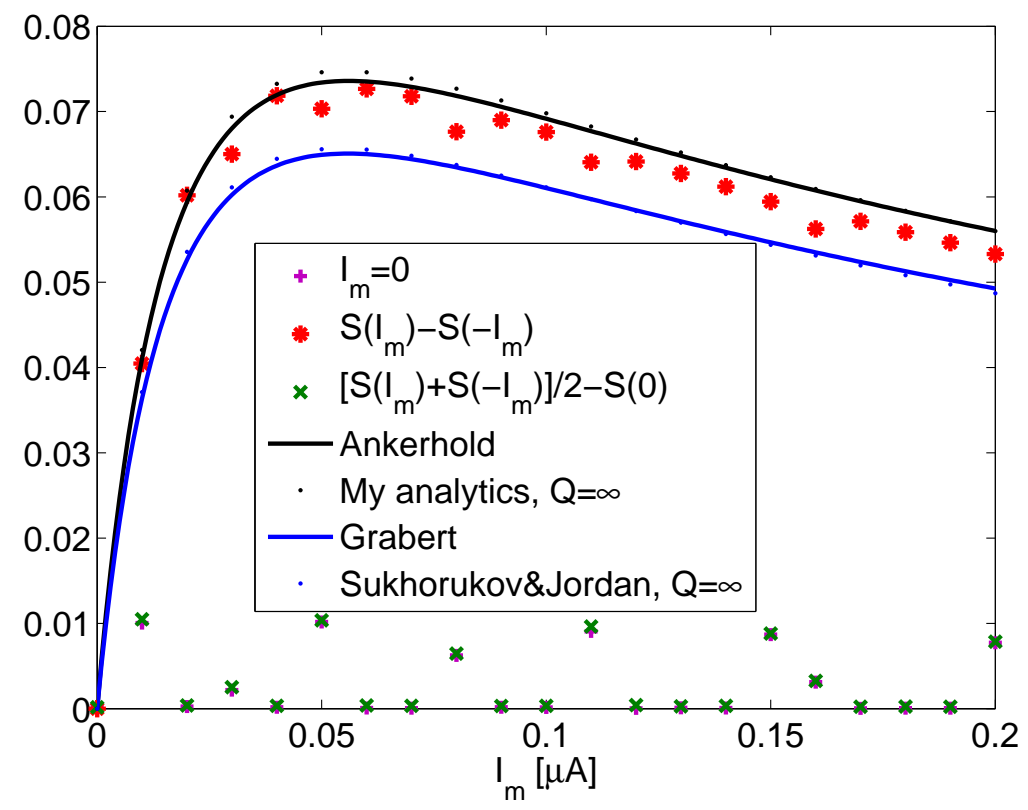

Figure 1. The logarithm of the escape rate asymmetry for temperature $T=$ $20 \mathrm{mK}$ and quality factor $Q=22$ corresponding to the Saclay experiment 10. For detailed explanation of various quantities and the values of other parameters see the main text.

by pluses which are essentially overlaid by crosses. The crosses show the quantity $\left[S\left(I_{m}\right)+S\left(-I_{m}\right)\right] / 2-S_{\text {anal }}(0)$ which probes the linearity of the calculated action in the third cumulant. If in the linear regime, the two polarities contain opposite contributions from the third cumulant which cancel in the sum and the subtracted action for zero third cumulant should nullify this quantity. In the second Fig. 2 with $Q=4$ this is indeed the case due to more stable numerics but one can see that those two control quantities are not strictly zero for the high-Q case. Their overlap, however, actually confirms the linear response regime. The deviation from zero of $\left[S\left(I_{m}\right)+S\left(-I_{m}\right)\right] / 2-S_{\text {anal }}(0)$ are solely due to the imprecision of the mean action without any influence of the third cumulant. This is further confirmed by the essentially regular behavior of the asymmetry $S\left(I_{m}\right)-S\left(-I_{m}\right)$. Moreover, it should be stressed that each point presents an independent calculation. Thus, the values of $I_{m}$ where the control quantities are zero as expected should be trustworthy regardless of the fact that the next value of $I_{m}$ may be calculated with insufficient precision. Moreover, the overall precision even in the $Q=22$ case is not catastrophically bad although it does not allow a fully reliable comparison with the concurrent theories.

The asymmetry $S\left(I_{m}\right)-S\left(-I_{m}\right)$ in Fig. 10 is compared with four different theories grouped into two sets (within the set they are virtually equal in the $Q \rightarrow \infty$ limit). It is (generalized numerical) evaluation $\grave{a}$ la Grabert 4 together with the result by Sukhorukov and Jordan [3] both of which predict basically $S\left(I_{m}\right)-S\left(-I_{m}\right) \propto 0.79(1-s)^{2}$ while the other set is my Eq. (9) and Ankerhold's [5] result $S\left(I_{m}\right)-S\left(-I_{m}\right) \propto 8 / 9(1-s)^{2} \doteq 0.89(1-s)^{2}$. While the difference in the predictions is only on the order of $10 \%$ and, thus, most likely irrelevant for experiments, 


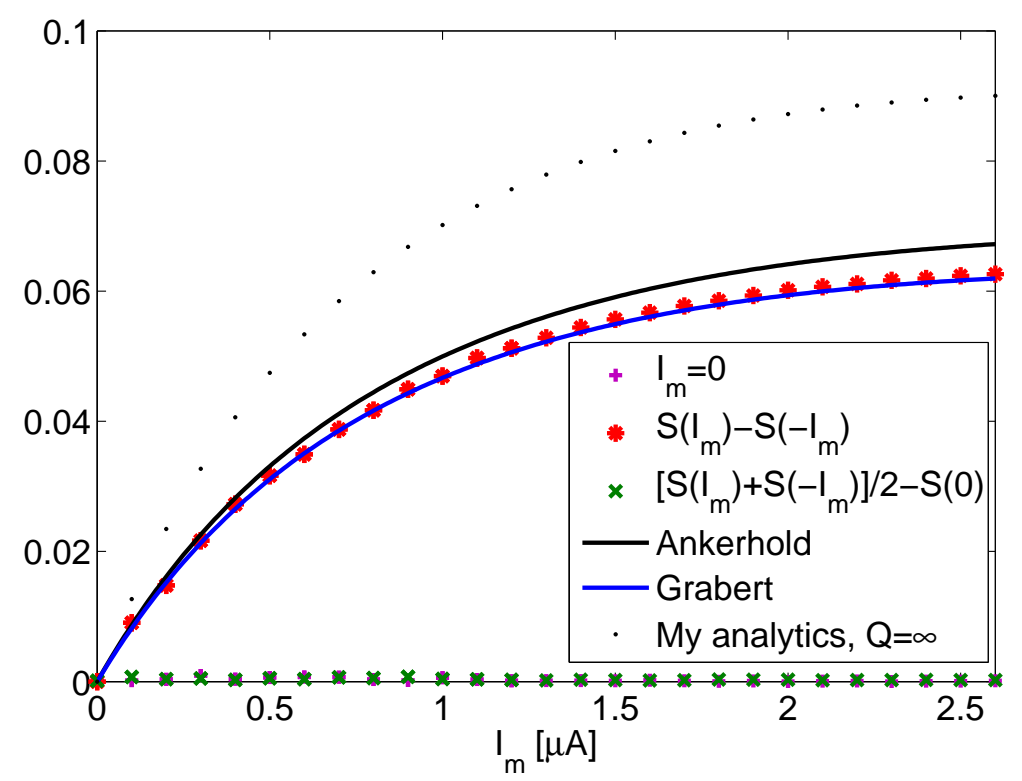

Figure 2. The logarithm of the escape rate asymmetry for temperature $T=$ $200 \mathrm{mK}$ and quality factor $Q=4$. For detailed explanation of various quantities and the values of other parameters, which are the same as in Fig. 1 and correspond to the Saclay experiment [10, see the main text.

it is relevant from a purely conceptual point of view which one is actually correct since it should help with the identification of possible misconceptions hidden in the failed approach(es). From the data presented in Fig. [1 it is clear that the more promising set is the Ankerhold-Novotný one. Despite of the scatter in the data, there are reliable points (where the control quantities turn into zero) which are closer to the 8/9-curve. The numerical calculation didn't use any linear perturbation theory or any approximation at all. The data are sheer results of the numerical evaluation of the BVP for general values of the parameters. The discrepancy of the data with the theoretical predictions may be caused by the finite, although rather high, value of $Q$. This can account for the difference between the numerics and 8/9-curve, however, it is inconsistent with Grabert's theory which predicts monotonic increase of the asymmetry with increasing $Q$, see Fig. 4 in Ref. 4]. Moreover, "Grabert's curve" was calculated for $Q=22$ even though it hardly deviates from its limiting $Q=\infty$ counterpart by SJ. Thus, the only salvation for the two theories 3, 4 could come from the numerics being wrong which is in principle possible but does not seem too plausible at this point.

If we now turn to the other Fig. 2 we see at the first place much better precision of the numerics as revealed by the control quantities being zero. The numerical data are again compared with Grabert's and Ankerhold's theories which provide alternatives for finite $Q$. I also show a curve for $Q=\infty$ to demonstrate significant deviations of the results for still relatively high $Q=4$ from the infinite- $Q$ limit. This should be remembered when interpreting experimental data of, e.g., the Helsinki group [19, 20, with $Q \approx 2.5$ via $Q=\infty$ theories. Ankerhold's theory (Eq. (13) of Ref. [5] with 
the 2/3-correction from the Erratum) is off the numerical data as well as Grabert's result thus clearly demonstrating only the interpolating status of this theory. The discrepancy is, however, rather small and, therefore, Ankerhold's formula seems to be a very cheap and efficient analytical interpolation scheme for an arbitrary $Q$.

Grabert's result on the other hand lies exactly on top of the numerical data in stark contrast to its apparent failure for the high- $Q$ case in Fig. 1 This is somewhat mysterious behavior which certainly deserves better understanding. What could go wrong in Grabert's reasoning? I have no clear answer to that, however, I do have a conjecture where there could be a problem hidden. Of course, I am fully aware that the problem could in fact be also in my numerics for $Q=22$ although its correspondence with my analytics represented by Eq. (9) is encouraging and not quite typical for bug-plagued numerics. Grabert's approach uses a straightforward perturbation theory at the level of trajectories connecting the fix-points. He argues that within the linear response in the third cumulant the equilibrium (unperturbed) solution is enough for evaluating the correction to the action. In more detail, provided the auxiliary Hamiltonian is split into equilibrium part and non-Gaussian perturbation $\mathcal{H}=\mathcal{H}_{\text {equil }}+\mathcal{H}_{3}$ the correction to the action reads $\Delta S_{3}=-\int_{-\infty}^{\infty} d t \mathcal{H}_{3}\left(y_{\text {equil }}(t)\right)$ (compare with his Eq. (77) in Ref. [4). This is analogous to the standard first order perturbation theory in quantum mechanics, the correction to energy is just the mean value of the Hamiltonian in the unperturbed state. However, one should recall that this formula is only applicable if the unperturbed state is non-degenerate. It's not obvious what is the analogous condition for classical trajectories, nevertheless, one may expect certain subtleties involved due to several conditions specific for the current problem. First of all, the BVP is formulated on an infinite time interval, there exists the continuous time shift symmetry, and the unstable/stable manifolds around the respective fix-points are two-dimensional (could this be the "degeneracy"?). The above formula for $\Delta S_{3}$ can be easily derived for finite time interval with fixed boundary conditions, however, can't the infinite time interval bring about omitted surface terms? I am quite sure these questions can be successfully handled by dynamical system theory experts.

\section{Experiment-related issues}

To this date (end of June 2008) there are two publicly available experimental results by the Helsinki group [19, 20, and by the Saclay group [10. The Helsinki experiment finds the asymmetry curve as a function of the measured current through the tunnel junction which has its shape in qualitative agreement with all previously mentioned theories (the $\sim 10 \%$ difference between different theories is undetectable at the level of precision of the experiment). However, the quantitative comparison with, e.g., Ankerhold's theory shows discrepancy on the order of $\sim 10$ (see the comparison in Ref. 20], recall the correction factor $2 / 3$ missing in that reference and further account for finite $Q=2.5$ contributing another factor of $1 / 2$ ). I haven't discussed the theory used by the Helsinki group for fitting the experiment since it's conceptually different from all the other discussed theories and I consider it to be semi-phenomenological with the prefactor (calculated in other theories) being adjusted to the experimental outcome, thus lacking a real predictive power. The other experiment by the Saclay group has been identified as most likely faulty due to a leak in the measurement circuit which prohibited the reliable determination of the bias current. Such an effect largely overshadows any asymmetry due to the third cumulant and, thus, no quantitatively 
reliable data are available from this experiment.

Regardless of this unsatisfactory status we may consider possible problems which are likely to be encountered, and maybe have already been encountered in the Helsinki experiment, when trying to compare the experimental outcome with theoretical predictions. The first issue is the one of the actual relevance of the exponential part of the rate asymmetry. Clearly, the experiment measures the rate asymmetry, not a theoretical concept of its exponential part. The rationale behind the dominance of the exponential part of the rate unfortunately doesn't necessarily carry over to the rate asymmetry, especially in the linear regime. The standard argument behind the dominance of the exponential part of (thermal) rates is that the large dimensionless barrier entering the exponent simply dominates the whole expression; moreover, the noise intensity (temperature) enters only the exponential part via the Boltzmann factor while the prefactor (attempt frequency) is temperature-independent. Now consider a weak noise with the third cumulant nonzero. This weak noise will supposedly weakly modify the rate. This will in general happen both through the exponent and the prefactor. In the linear response regime in the third cumulant the correction in the exponent can be safely expanded and the resulting linear correction will add to the linear correction stemming from the prefactor. At this stage there is no a priori difference between these two contributions. Of course, in practice one of them (presumably the prefactor part) can still be negligible. What are the prospects for this to happen? We have seen that in the realistic setup studied in the previous section the asymmetry due to the exponential part of the rate reaches values on the order of $\sim 10 \%$ at maximum. The expected correction due to the prefactor is of the form $k_{B} T_{\text {eff }} / E_{J} \cdot I_{m} / I_{0}$. The first factor, dimensionless temperature, is of the order of $\sim 1 \%$ while the other factor, dimensionless measured current, is of the order of $\sim 1$. Thus, in total, we have an effect of the order of $\sim 1 \%$ which can be, depending on the actual numerical prefactor, comparable to the exponential part. This somewhat pessimistic scenario can be further supported both by the discrepancy found in the Helsinki experiment as well as by the mismatch between Ankerhold's theory and direct stochastic simulations performed in connection with the Saclay experiment in Ref. [10] (see their Fig. 7a) where a multiplicative factor of 2 difference was found for the dimensionless barrier height $\sim 6$. While this value lies at the border of reliability of the WKB approach and corrections for larger barriers (especially the experimentally relevant one 10.4) may be expected, they are not expected to be of order of $100 \%$. Therefore, it seems that the asymmetry stemming from the prefactor may be relevant for experiments. This is a rather bad news for theoreticians since the calculation of the prefactor for non-equilibrium rates is an involved task, see the discussion in Ref. [7] and references therein.

So we finally come to the question whether one can achieve a nonlinear regime with underdamped JJs. The problem is apparently in the fact that the effective temperature raises with the measured current in such a way that it simply dominates the escape mechanism and corrections due to higher order cumulants are just negligible. This is clearly reflected in the plot in Fig. 1 where the originally growing (with $I_{m}$ ) curve for small $I_{m}$ eventually bends downwards again for larger $I_{m}$. While the first part is governed by the third cumulant growing with $I_{m}$, the declining part corresponds to the case when the contribution of $I_{m}$ to the effective temperature beats the raising third cumulant. The same effect would be seen in Fig. 2 for larger values of $I_{m}$. This behavior could be diminished by weakening the effect of the measured current on the effective temperature, see the expression for the effective temperature. This 
should be achieved by decreasing the value of the effective shunt resistance $R$. This, in turn, would imply decreasing quality factor which seems experimentally unacceptable beyond the point when the switching ceases to exist and only phase diffusion is present. The quality factor thus should be maintained at a reasonably high value which can be achieved by increasing capacitance $C$. That in turn will decrease the third cumulant contribution via the formula preceding Eq. (9). At this point the problem turns into a bad joke. There may be, however, a parameter window where a subtle compromise can be achieved. This should be seriously considered by carefully examining different parameter dependencies and testing experimentally acceptable numbers.

\section{Conclusions}

In this work I have reviewed in detail the status of the problem of the measurement of the Full Counting Statistics by the switching dynamics of an underdamped Josephson junction. I have presented a general theory for the weak noise based on the WKBlike approximation and calculated the rate asymmetry due to a weak third cumulant analytically in the limit of very high quality factor of the junction. This calculation has been critically compared to other theories and their possible shortcomings have been identified and pointed out. Further, I have developed a numerical scheme for solving the boundary value problem determining the exponential part of the non-equilibrium escape rate under general circumstances, i.e. beyond the linear perturbation theory. Using this scheme I have calculated the exponential part of the rate asymmetry for experimentally relevant set of parameters and compared the findings with various linear theories. Again, this helped with the identification of the status of concurrent theories. Eventually, I have briefly discussed issues related to the interpretation of present and future experiments, in particular the question of the relevance of the rate exponential prefactor for the rate asymmetry and the feasibility of achieving the nonlinear regime.

There are plenty of unsolved problems and open issues within this field of research. Starting with those more particular and technical, it would be rewarding to fully clarify the status of concurrent theories, in particular that by Grabert which performs amazingly well for intermediate range of $Q$ 's while it seems to fail for large values of $Q$. Although the discrepancy is not too large, Grabert's theory is supposed to work in that regime as well and, thus, the discrepancy raises serious questions about its very foundation. On the other hand, it would be very helpful to further develop and fully stabilize (if possible) my numerical scheme used for the solution of the BVP. If this attempt were successful the numerical code could be used for interpreting future experiments routinely since it is very fast and efficient as long as it converges which unfortunately occasionally doesn't happen. On a more general level, it should be further studied what is the effect of nominally subleading terms in the rate on the rate asymmetry. It appears that the conventional arguments for the dominance of the exponential part of the rate may not be applicable to the rate asymmetry, especially in the linear regime. And last but not least, a most important question whether one can actually use underdamped Josephson junctions for the measurement of the whole FCS and not only the third cumulant in the linear regime is still open and waiting for final answer which, if affirmative, could bring the field of the FCS to new milestones. 


\section{Acknowledgments}

I am grateful to P. Hänggi, I. Khovanov, K. Netočný, and P. Talkner for useful and stimulating discussions, continuous encouragement, and for pointing out relevant references. I acknowledge the support by the grant 202/07/J051 of the Czech Science Foundation. This work is also a part of the research plan MSM 0021620834 financed by the Ministry of Education of the Czech Republic.

Post-acceptance note. After the acceptance of this manuscript with minor corrections I became aware of a comment arXiv:0807.2675 by Sukhorukov and Jordan. Although at this point I am unable to decide whether that comment really settles the above mentioned discrepancy between our theories, I recommend the interested reader to check out their paper for an independent point of view on the issue.

[1] J. Tobiska and Yu. V. Nazarov, Phys. Rev. Lett. 93, 106801 (2004).

[2] J. P. Pekola, Phys. Rev. Lett. 93, 206601 (2004).

[3] E. V. Sukhorukov and A. N. Jordan, Phys. Rev. Lett. 98, 136803 (2007).

[4] H. Grabert, Phys. Rev. B 77, 205315 (2008).

[5] J. Ankerhold, Phys. Rev. Lett. 98, 036601 (2007). Also see erratum Phys. Rev. Lett. 99, 139901 (2007).

[6] J. Kevorkian and J. D. Cole, Multiple Scale and Singular Perturbation Methods. Springer, Berlin, 1996.

[7] P. Hänggi, P. Talkner, and M. Borkovec, Rev. Mod. Phys. 62, 251 (1990).

[8] H. Risken, The Fokker-Planck Equation. Springer, Berlin, 1989.

[9] J. P. Pekola et al., Phys. Rev. Lett. 95, 197004 (2005).

[10] B. Huard et al., Annalen der Physik, 16(10-11), 736 (2007).

[11] A. Kamenev, Many-body theory of non-equilibrium systems (2004); cond-mat/0412296

[12] R. Graham. Macroscopic potentials, bifurcations and noise in dissipative systems. In Frank Moss and P. V. E. McClintock, editors, Theory of continuous Fokker-Planck systems, volume 1 of Noise in non-linear dynamical systems, chapter 7, page 225. Cambridge University Press, 1989.

[13] S. Beri et al., Phys. Rev. E 72, 036131 (2005).

[14] L. F. Shampine, I. Gladwell, and S. Thompson, Solving ODEs with Matlab. Cambridge University Press, 2003.

[15] W. H. Press, B. P. Flannery, S. A. Teukolsky, and W. T. Vetterling, Numerical Recipes in FORTRAN 77: The Art of Scientific Computing. Cambridge University Press, second edition, 1992.

[16] Oleg Kogan. Scaling crossovers in activated escape of nonequilibrium systems: a resonantly driven oscillator; arXiv:0805.0972 v1, May 2008.

[17] D. G. Luchinsky et al., International Journal of Bifurcation and Chaos, 12(3), 583 (2002).

[18] I. A. Khovanov et al., Phys. Rev. Lett. 96, 083903 (2006).

[19] A.V. Timofeev et al., Phys. Rev. Lett. 98, 207001 (2007).

[20] J.T. Peltonen et al., Physica E 40, 111 (2007). 